\title{
Pratique Raisonnée de la Langue et exercices
}

Reasoned practice of language and exercices

Jean-Pierre Gabilan

\section{(2) OpenEdition}

\section{Journals}

\section{Édition électronique}

URL : https://journals.openedition.org/dhfles/6932

DOI : $10.4000 /$ dhfles. 6932

ISSN : 2221-4038

\section{Éditeur}

Société Internationale pour l'Histoire du Français Langue Étrangère ou Seconde

Édition imprimée

Date de publication : 1 décembre 2019

Pagination : 473-495

ISBN : 0992-7654

ISSN : 0992-7654

Référence électronique

Jean-Pierre Gabilan, « Pratique Raisonnée de la Langue et exercices », Documents pour l'histoire du français langue étrangère ou seconde [En ligne], 62-63 | 2019, mis en ligne le 01 janvier 2020, consulté le 27 mars 2023. URL : http://journals.openedition.org/dhfles/6932 ; DOI : https://doi.org/10.4000/ dhfles.6932

Ce document a été généré automatiquement le 27 mars 2023.

Tous droits réservés 


\title{
Pratique Raisonnée de la Langue et exercices
}

\author{
Reasoned practice of language and exercices
}

Jean-Pierre Gabilan

\section{Introduction}

1 Une étude conduite en 2002 dans huit pays européens visant à évaluer les compétences en anglais d'élèves de quinze ans révéla que les performances des élèves français étaient nettement inférieures à celles des élèves des sept autres pays participants. Le rapport publié par l'Éducation Nationale ${ }^{1}$ montrait, entre autres, une faiblesse inquiétante pour la compréhension de l'oral $^{2}$. Le rapport faisait également état des pratiques des enseignants et il ressortait que pour les enseignants français, "le préalable à l'apprentissage d'une langue reste la connaissance de la grammaire». Il était précisé dans la foulée que «l'enseignant français développe une recherche de la perfection qui peut inhiber les élèves ». Sans aborder un domaine où la seule psychologie de l'élève serait au centre du débat, nous voudrions dans les pages qui suivent faire un état des lieux de l'enseignement de cette grammaire dont on regrettait en 2002 que les enseignants la considèrent comme un préalable à l'apprentissage. Ce que ne disait pas directement ce rapport, et ce dont les rédacteurs n'avaient sans doute pas conscience, c'est qu'il y a grammaire et grammaire. Ce qui est appelé grammaire par certains, et sans doute par une majorité, est en fait un ensemble de bouts de ficelle, de trucs et de combines qui sont bien éloignés de la réalité de fonctionnement des langues en général, de la grammaire au sens fort. Quelle est la grammaire enseignée aujourd'hui dans les collèges et lycées ${ }^{3}$ de France ? Où en est la Pratique Raisonnée de la Langue prônée en 1985 dans les textes officiels qui avait suscité beaucoup d'espoir et d'engouement chez les linguistes? 


\section{La Pratique Raisonnée de Langue : naissance et disparition}

2 D'abord passées inaperçues, les instructions officielles de $1985^{4}$ furent à l'origine d'un sigle, PRL, qui s'est très rapidement substitué au mot "grammaire». Ce sigle était apparu dans le texte officiel de la façon suivante :

[...] Grâce à la pratique raisonnée de la langue, l'élève atteint un troisième objectif. Il exerce sa réflexion sur les grands principes de fonctionnement de l'anglais : sa compétence implicite en langue maternelle s'en trouve partiellement explicitée et l'appropriation facilitée. [...] (ministère de l'Éducation nationale 1985 :114)

On sait aujourd'hui qu'en 1985 ces quelques mots ne pouvaient être compris du public visé, et donc des professeurs en exercice ${ }^{5}$. Qui en effet, dans l'enseignement secondaire, aurait pu définir avec précision ce que l'on entendait par «grands principes de fonctionnement de l'anglais" ? Si ici où là des chercheurs avançaient que l'anglais, comme n'importe quelle autre langue, était régi par des "grands principes de fonctionnement", peu de manuels de grammaire publiés en faisaient état ${ }^{6}$. Une consultation des manuels d'anglais en vigueur dans la deuxième moitié des années quatre-vingts permet d'être très assertif sur ce point: les «grands principes de fonctionnement de l'anglais " n'avaient pas retenu l'attention des concepteurs des ouvrages en question. On est en droit de s'interroger, plus de trois décennies plus tard, sur la raison de cet état de fait: pourquoi avait-il été décidé en 1985 de tenir pour acquis l'existence de "grands principes de fonctionnement", alors que les professeurs n'avaient dans leur majorité reçu aucune formation linguistique digne de ce nom ? Ce qui aujourd'hui encore est loin d'être entré dans les mœurs ne pouvait que rester lettre morte à l'époque, faute d'être compris par le public même auquel les instructions officielles étaient destinées. Le complément d'informations publié deux ans plus tard dans le Programme pour la classe de seconde reprenait le texte de 1985 sous une forme plus développée, mais finalement aussi opaque :

La démarche de conceptualisation permet à l'élève de relier les données disparates de surface à un système cohérent. [...] L'élève peut être amené à percevoir certains aspects de la cohérence interne de la langue qu'il étudie [B.O.E.N. nº spécial 3 du 9 juillet 1987 (classe de seconde), 19]

4 Ce que certains linguistes affirmaient depuis longtemps ${ }^{7}$ - sans pour autant le montrer de façon convaincante - ne pouvait toujours pas être compris tel quel en 1987 pour pouvoir être intégré dans l'enseignement. Aucune des deux ou trois collections de manuels de lycée publiées après 1987, à l'instar des collections antérieures, ne tenta de mettre en avant le moindre système organisateur; ceci en dit long sur le degré de pénétration de concepts qui étaient censés - et auraient dû - connaître un plus grand succès. Et en amont, les sempiternels trois mots soulignés dans un texte ${ }^{8}$ pour évaluer la compétence grammaticale des candidats au CAPES ne pouvaient à eux seuls être porteurs d'avenir ; quel candidat passerait en effet des heures à travailler une épreuve de quelques minutes, et n'étant pas de surcroît dotée d'un fort coefficient ${ }^{9}$ ? Il est assez surprenant d'ailleurs que ce qui constitue la base de tout enseignement de langue n'ait jamais été reconnu par le concours de recrutement.

Les "grands principes de fonctionnement de l'anglais » n'eurent donc pas l'heur de retenir l'attention de la profession, et ce malgré quelques effets d'annonce assez tapageurs, mais non suivis d'effet. C'est ainsi que dans le guide pédagogique d'un 
manuel de collège publié en $1994^{10}$ - près de dix ans après la publication des textes officiels de 1985 donc - les auteurs parlaient d'une "grammaire moderne " et prétendaient avoir procédé à une "grande lessive ». Certes, les grands noms étaient cités et l'on exhortait les professeurs à « s'ouvrir aux théories d'Antoine Culioli, d'Henri Adamczewski, de Michael Halliday, de Gustave Guillaume (via André Joly) et à ne pas négliger ce qui permet d'aider l'enfant à maîtriser et à s'approprier la langue. » Une consultation du manuel de l'élève (leçons et pages de grammaire) permettait rapidement de comprendre que de grande lessive il n'y avait pas eu. Sur le seul point sensible des deux présents de l'anglais ${ }^{11}$, les actions habituelles - rebaptisées "événements stables" - étaient opposées à " ce qui se passe en ce moment ". Comme dans la collection Imagine You're English ${ }^{12}$ du milieu des années soixante-dix, on opposait ensuite les deux énoncés suivants: Matt wears a uniform at school. // He isn't wearing a uniform today. It's Sunday!

6 La révolution grammaticale annoncée n'était pas au rendez-vous et on peut légitimement s'étonner du décalage entre les prises de position et les leçons effectivement proposées aux élèves et à leurs professeurs. Il en est souvent ainsi dans l'enseignement de l'anglais où les mots "système ", "cohérence ", " autonomie ", et " prise de parole en continu » sont quelque peu détournés de leur sens premier. Ce qu'il est advenu de la PRL à l'échelle du pays est à l'image de la démarche que les auteurs du manuel cité ci-dessus avaient adoptée.

7 L'adjectif « raisonné(e) » n'était pas en 1985 une nouveauté pour la communauté des anglicistes. Denis Girard ${ }^{13}$ avait publié en 1978 une Grammaire raisonnée de l'anglais et annonçait dans la préface son souci pédagogique d'imposer « l'éclectisme et le recours à diverses théories linguistiques. » La Pratique Raisonnée de la Langue esquissée en 1985 allait au-delà, et était accompagnée de la « réflexion sur les grands principes de fonctionnement de l'anglais ", la langue maternelle étant également concernée. Pour que pratique raisonnée il y eût, encore eût-il fallu que l'on sache ce sur quoi il fallait exercer la réflexion en question. Or la réflexion grammaticale de l'époque - et les manuels de grammaire à forte diffusion et autres livres de cours le montrent bien était à des années-lumière de toute réflexion au sens fort, de toute pratique raisonnée donc. Quelle réflexion en effet mener à bien face à des règles de grammaire faisant appel aux " actions", aux « centimètres $»^{14}$, à " l'insistance sur la durée », etc. ? Pour intéressant et hardi qu'ait pu être cet encouragement à adopter un autre type de regard sur le fonctionnement de la langue, les concepteurs (anonymes à ce jour) du texte de 1985 avaient pour ainsi dire jeté une bouteille à la mer, et il n'est pas assuré qu'ils en aient attendu quoi que ce soit en retour. Il est d'ailleurs assez révélateur de constater que les tableaux qui suivaient ces quelques lignes d'introduction avaient une entrée de type notion/fonction ${ }^{15}$; de nouvelle grammaire, dans le document officiel même, il n'y avait donc point. De façon assez symptomatique, de Pratique Raisonnée de la Langue on est passé à PRL, et le sigle a contribué à faire oublier chacune des trois composantes de cette étiquette ${ }^{16}$. Pire, pour de nombreux professeurs qui ont cru à une nouvelle mode pédagogique - encore une? - la question était de savoir, de façon beaucoup plus pragmatique, à quel moment du cours il fallait « faire la PRL ». Mais à aucun moment la question du réel contenu de cette démarche n'était abordée. L'étude du "point de grammaire » était déjà une composante du cours traditionnel, et les professeurs qui avaient connu le CAPES pratique ${ }^{17}$ à l'ancienne étaient familiers de la découpe du cours : un peu de grammaire, un peu de traduction, un peu de lecture, etc. le tout sous le contrôle du chronomètre. Donc PRL et grammaire traditionnelle ont été confondues, 
et il ne pouvait en être autrement. On peut donc dire qu'entre 1985 et 1995, date de publication de nouveaux programmes pour la classe de sixième, la situation grammaticale est restée identique à ce qu'elle avait toujours été. Les programmes de collège publiés classe par classe entre 1995 et 1998 n'ont pas repris le sigle PRL. La phonologie, qui avait fait son entrée dans le texte de $1987^{18}$, semblait singulièrement renforcée ${ }^{19}$. Le programme grammatical, selon la classe visée, se caractérisait par l'éclectisme, approche positive pour les uns, plutôt négative pour les autres quand on sait que les deux ou trois théories linguistiques présentes en France ne sont pas réconciliables tant leurs fondements théoriques sont différents. Une fois encore, les éléments théoriques parfois sollicités ne pouvaient être compris tels quels par un corps professoral non préparé. À la même période, les éditeurs scolaires crurent utile de se lancer de concert à l'assaut du marché potentiellement lucratif que représente le collège. C'est ainsi qu'en 1994, pas moins de sept manuels de sixième ${ }^{20}$ vinrent s'entasser dans les casiers des professeurs de collège. Ces ouvrages, tous publiés un an avant la parution des "nouveaux » programmes, furent un an plus tard estampillés " conforme aux nouveaux programmes" par les éditeurs, ce qui en disait long sur la nouveauté des programmes en question. Dix ans après la PRL, les sept manuels parus avaient adopté, pour certains d'entre eux, de nouvelles étiquettes. Le "présent progressif » cédait la place à "présent en -ing » ou «présent be-ing» - mais trois de ces manuels conservaient "présent progressif» ou "présent continu». Quelles que soient les étiquettes retenues, les analyses restaient toutefois conformes à l'approche traditionnelle, et aux habitudes on opposait les arrêts sur image ou les actions en cours, comme le faisait déjà L'Anglais Vivant de Carpentier-Fialip (1931) un peu de plus de soixante ans auparavant. Pourquoi dans ces conditions publier de nouveaux manuels ${ }^{21}$ ? Parallèlement, une Grammaire raisonnée au lycée paraissait en 1994 (Persec), reprenant donc dans son titre une expression devenue "porteuse ». Une lecture minutieuse du contenu de l'ouvrage ne permettait pas de trouver la moindre référence au concept emprunté dans le titre. Une fois encore était à l'œuvre le principe selon lequel les annonces ne sont pas suivies d'effets. Citons encore un ouvrage paru en 2000 (Martina) $^{22}$ dont l'introduction, prometteuse, pouvait donner quelque espoir. Le passage suivant est assez représentatif :

Adapter un savoir universitaire. Le pas à franchir n'est pas si naturel. Prenons-en pour exemple le «lapsus» de "présent continu » qui apparait parfois dans les copies de didactique rédigées par les étudiants au CAPES pourtant aguerris à la grammaire énonciative. C'est que les habitudes sont tenaces et qu'il n'est pas facile de faire abstraction de la façon dont on a soi-même appris la grammaire anglaise dans l'enseignement secondaire. (Martina $2000: 11$ )

Voici deux exemples de tableaux (p. 30 et p. 51) que présente l'ouvrage en question : dans les deux cas les activités qui découlent de ces tableaux ont pour cible des élèves de classe de sixième :

Martina $(2000: 30)$

INTRODUCTION DU PRESENT SIMPLE EN $6^{\mathrm{e}}$ AUX PERSONNES AUTRES QUE HE/SHE

Que faire découvrir?

- Le présent simple est une forme qui n'a pas recours à un auxiliaire.

- Il n'y a pas de rapport avec le moment de l'énonciation mais au contraire rupture avec le moment où l'on parle.

- L'énonciateur présente un fait sans le commenter. 
- Valeurs du présent simple ; présent de généralité :

caractéristique du sujet ;

habitude : on parlera du présent de routine ;

fait général.

- Aux formes interrogative et négative, il a recours à l'opérateur do.

- Le français ne fait pas cette distinction entre le présent spécifique, qui décrit une situation particulière, et le présent de généralité.

Martina (2000: 51)

PRESENT BE+-ING :

CONTRASTE AVEC LE PRESENT SIMPLE

Que faire découvrir?

- L'anglais a recours à deux formes différentes au présent : le présent simple et le présent en be+-ING.

- Be+-ING est une forme liée qui signale un rapport avec le moment de l'énonciation.

- Cette forme est composée de deux éléments.

- Be+-ING est un aspect.

- Valeurs de Be+-ING :

- situation particulière et non générale ;

- valeur d'inaccompli ;

- valeur de visualisation : on peut parler d'arrêt sur image ;

- valeur de commentaire : on s'intéresse à l'activité du sujet ; l'énonciateur commente cette activité.

Les "vieilles" habitudes fustigées dans l'introduction, et ailleurs dans l'ouvrage, semblaient ne pas avoir épargné l'auteur lui-même car on retrouve là ce qui avait toujours été enseigné à propos des deux présents de l'anglais. Certes, le mot " aspect » était lâché, et avec lui, en introduction de l'ouvrage, "grammaire énonciative ». Mais opposer le général au particulier n'était quand même pas une nouveauté dans l'histoire de l'enseignement de l'anglais. Pour qui aurait voulu trouver des raisons pour réfuter l'introduction de l'approche dite énonciative dans l'enseignement de l'anglais ${ }^{23}, \mathrm{La}$ Grammaire de l'Anglais au Collège offrait des arguments de poids. Et si de fait, la grammaire énonciative et ses partisans préfèrent "valeur d'inaccompli » à "action inachevée " et "rupture avec le moment d'énonciation " à "généralité ", il est vraiment à craindre que l'on ne puisse jamais enseigner l'anglais tel que les anglophones le parlent. Le "temps » appelé Présent simple est tout à fait approprié dans des situations non générales et bel et bien ancrées dans une situation spécifique liée au moment de parole. Il suffira ici de citer : You time starts ... now ! qui est prononcé par tout animateur de jeux télévisés ou radiophoniques. Il est impossible et donc agrammatical d'avoir recours à be+ing ici. Il est tout à fait possible par ailleurs d'avoir recours à be+ing dans des énoncés qui dénotent une généralité, une récurrence, une situation stable. La presse offre des occurrences telles que: Children with type 1 diabetes are not receiving proper treatment in schools (ITV News, 2 juin 2015).

Il est bel et bien question de faits récurrents, d'une situation générale. Il ne sera pas utile ici de multiplier les contre-exemples aux règles énoncés plus haut ${ }^{24}$.

L'importance qui semblait être attachée à la grammaire dans les textes de 1985, et ce en raison d'une entrée mal préparée comme nous l'avons expliqué, donna lieu à différentes réactions. Il y eut bien ici et là dans quelques académies des formations parfois organisées par le biais de la M.A.F.P.E.N.et des C.R.D.P ${ }^{25}$ mais les professeurs ne virent, dans leur majorité qu'une nouvelle lubie pédagogique. En 2002, de nouveaux textes faisaient singulièrement machine arrière :

L'apprentissage grammatical exige à la fois une pratique régulière de la langue et une réflexion sur celle-ci. L'analyse grammaticale apporte un éclairage utile, parfois indispensable, sur le fonctionnement de la langue mais ne saurait être une fin en soi. (B.O.E.N. $n^{\circ} 7$ hors-série du 3 octobre $2002: 14$ ) 
On ne peut qu'être surpris par la mention " parfois indispensable » qui fut insérée dans ce document officiel. Sans doute était-il révélateur d'une attitude générale face à la grammaire au moment où le $\mathrm{CECRL}^{26}$ entrait en vigueur, changeant singulièrement les objectifs d'enseignement. Introduite de façon inopportune, à un moment de l'histoire de la réflexion sur la langue encore mal affirmé, la Pratique Raisonnée de la Langue était mort-née au moment même où les textes la faisaient naître.

\section{Les grands principes de fonctionnement de l'anglais}

Affirmer l'existence de grands principes de fonctionnement d'une langue, voire des langues en général, requiert en amont une approche de la grammaire qui dépasse le stade des observables. "Grands principes " signifie aussi que ces principes sont en nombre limité. La grammaire telle que la connaît le grand public ne repose pas sur une telle approche, et chaque nouveau chapitre est abordé sans que l'étude du précédent soit d'un quelconque secours ${ }^{27}$. De même, le type de grammaire qui est utilisé pour enseigner la grammaire de leur langue maternelle aux francophones n'est pas réutilisable pour aborder l'anglais. Une grammaire dont les principes reposent entre autres sur une théorie permettant de savoir comment accorder le participe passé n'est pas transférable à une autre langue. Toute langue est construite sur un réseau d'oppositions. Ce que la phonologie a montré est transférable à la grammaire. Ainsi, en réfléchissant en termes d'oppositions, on parvient à dresser une liste d'opposables régis par un même principe, un même vecteur; c'est sans doute ce que le texte de 1985 signifiait, mais sans précision.

\section{Le concept de statut}

Dès lors qu'il y a choix à effectuer il y a sens. Un des mots-clés dans cette optique est le concept de "statut». Dans les deux énoncés suivants: I need a car // I need the car. Le nom car est «saisi » dans l'énoncé de deux façons différentes : d'abord par le biais de l'article $a$ puis par le biais de l'article the. Dire que $a$ est un article « indéfini » et the un article « défini $\aleph^{28}$ est certes intéressant mais insuffisant. Avec a l'énonciateur confère à car un statut posé - ou statut assertif. L'énonciateur a choisi un item, car, dans une liste infinie, d'où son assertivité. Avec the l'énonciateur considère que le co-énonciateur a déjà identifié l'item car, d'où son statut repris ou non assertif. L'article a n'existe que parce que the lui fait pendant. On peut à juste titre parler d'un "grand principe de fonctionnement » qui régit le choix de l'article en anglais - et dans toutes les langues qui possèdent un système comparable.

\section{Le concept de statut dans le système des «temps »}

Ce qui est saillant en anglais c'est dans un premier temps l'opposition simple vs be+ing, ce qui donne deux « temps ${ }^{29}$ » de base et huit assemblages possibles :

\begin{tabular}{|l|l|l|}
\hline & simple & be+ing \\
\hline PRESENT & He plays football & He is playing football \\
\hline
\end{tabular}




\begin{tabular}{|l|l|l|}
\hline $\begin{array}{l}\text { PRESENT } \\
\text { [have + participe passé] }\end{array}$ & He has played football & He has been playing football \\
\hline PRETERIT & He played football & He was playing football \\
\hline $\begin{array}{l}\text { PRETERIT } \\
\text { [have + participe passé }\end{array}$ & He had played football & He had been playing football \\
\hline
\end{tabular}

La même opposition binaire affecte le système des modaux. Voici quelques exemples :

\begin{tabular}{|l|l|l|}
\hline & sans be+ing & avec be+ing \\
\hline WILL & He will play football & He will be playing football \\
\hline MUST & He must play football & He must be playing football \\
\hline SHOULD & He should play football & He should be playing football \\
\hline
\end{tabular}

Là où la tradition a établi un lien direct entre le réel (l'extralinguistique) et la grammaire, une approche fondée sur le statut des composantes offre des analyses fondées sur la grammaire. En guise d'illustration, voici un énoncé vu dans un magasin de la chaîne britannique Marks and Spencer: "When you buy our cotton products you are helping us improve cotton farming globally. » Une analyse menée dans les termes de la tradition - action « habituelle » versus action « en train de » - est inopérante. L'énoncé est agencé comme suit: dans un premier temps l'énonciateur pose des données, nomme ce que fait le sujet you: énoncé dynamique de statut posé/assertif. Puis l'énonciateur analyse ce premier énoncé et indique dans quelle situation se trouve alors le «sujet acheteur de produits en coton». Ceci explique le recours au verbe be - are puis à une nominalisation en -ing: [help us improve cotton farming globally]. «You buy our cotton products = ing [help us improve cotton farming globally] ». Le statut du bloc dominé par -ing est dès lors tout à fait différent de la première partie de l'énoncé.

En résumé : When + you + buy + our cotton products : statut posé/assertif du verbe puis du complément; you are [helping us improve cotton farming globally] : statut repris/non assertif du bloc dominé par -ing. Le statut du sujet you change également: de sujet « agentif » il devient sujet « objet de discours ${ }^{30}$ ».

Quant à l'opposition du type: He will visit his friend on Sunday // He will be visiting his friend on Sunday, elle ne peut qu'être comprise qu'en termes de statut : «He will visit his friend on Sunday. », annonce la probabilité d'une visite effectuée dimanche - la date est posée. « He will be visiting his friend on Sunday. ", a pour but d'utiliser un fait acquis la visite prévue dimanche - dans un contexte tel que le suivant : «But Peter will not be here with us! He will be visiting his friend on Sunday. Dans cet exemple, [visit-his-friendon Sunday] est ce qui permet de corroborer ce qui a été dit au préalable, le point d'appui forcément antérieur dans la chronologie des opérations de mise en discours à l'énoncé qui arrive en premier dans la chaîne parlée. 


\section{Le concept de statut pour rendre compte du rôle joué par $D 0^{31}$}

\begin{tabular}{|l|l|l|}
\hline & $\varnothing$ & DO \\
\hline PRESENT & He plays football & He does play football/He does NOT play football \\
\hline PRETERIT & He played football & He did play football/He did NOT play football \\
\hline
\end{tabular}

21 Il faut aller bien au-delà de l'expression d'une insistance pour rendre compte de la structuration des énoncés de type affirmatif comportant DO. Il faut plutôt parler de « confirmation » et comme on ne peut confirmer que ce qui a été déjà été posé, on comprend dès lors que le rôle de $D O$, certes porteur d'un accent tonique dans les cas de conflit ou de reprise forte, est là avant tout pour coder le statut repris et donc non assertif de la relation he/play football. Les énoncés négatifs reçoivent la même analyse à la différence que NOT annule ce qui a été posé au préalable. Que l'on dise «DO come in! » ou «DON'T come in! », dans les deux cas l'interlocuteur est sur le pas de la porte. Le vecteur statut posé/statut repris est encore à l'œuvre. Dans des énoncés construits sur le modèle suivant : Not only did he spend all his money but he also borrowed some from his friends; le rôle de did est de coder que la relation he/spend all his money est de statut repris/non assertif. L'énonciateur n'affirme plus la relation; il l'évoque pour servir de base à ce qui suit et qui est de statut posé/assertif.

\section{Autres illustrations du concept de statut}

On ne peut pas ne pas rencontrer les oppositions qui suivent en abordant l'anglais ${ }^{32}$ :

- to/-ing : He likes to play football // He likes playing football.

- some/any : Do you need some money? // Do you need any money?

- this/that : You should take this key // You should take that key.

- nearly/almost : I am nearly 15 // I am almost 15.

- of /'s : the wife of the Belgian ambassador // the Belgian ambassador's wife.

- little - few // a little - a few : I have little money left - I have few postcards left // I need a little money - I need a few postcards.

La formation linguistique des futurs professeurs d'anglais n'étant souvent pas à la hauteur de la tâche les regroupements proposés ci-dessus ne peuvent, la plupart du temps, être appréhendés convenablement. Le «mot de la présidente» du jury de CAPES, dans le rapport de la session de $2017^{33}$ est révélateur :

L'exercice de réflexion sur la langue continue de poser les plus grands défis à de nombreux candidats. La faiblesse des résultats globalement obtenus à cette partie de la seconde épreuve conduit à rappeler, cette année encore, l'importance pour un futur enseignant de savoir expliciter et analyser le fonctionnement de la langue cible à ses élèves. [...] Tout candidat raisonnable mesure par ailleurs que de solides acquis en linguistique peuvent être réinvestis avec bonheur intellectuel et profit immédiat dans l'analyse d'un texte littéraire ou d'un document de civilisation. De plus, il est impossible de concevoir une réflexion didactique ou une séquence pédagogique qui ne soit pas ancrée dans une solide base de connaissances des mécanismes de fonctionnement des deux langues. La connaissance de la langue et la capacité à mobiliser ses connaissances universitaires pour expliquer son 
fonctionnement sont autant de caractéristiques attendues chez un enseignant

(Manes-Bonnisseau 2017 : 11).

24 On ne sera pas surpris de lire que pour bon nombre de candidats, plus de trente ans après la publication des textes de 1985, une épreuve de réflexion sur la langue représente un défi. Mais on peut et on doit le déplorer.

\section{Grammaire et exercices - d'abord pour les professeurs}

La tradition grammaticale nous a légué un couple, "grammaire et exercices » qui, pour beaucoup, à l'instar de "orthographe-grammaire", est tout aussi indissociable. Cette association demande à être revue et corrigée. Les exercices sont finalement très révélateurs de la conception du fonctionnement de la langue qu'ont les auteurs des exercices en question.

La notion même d'exercice est à reconsidérer car, pour le domaine des «temps " par exemple, les éternels "mettre le verbe au temps qui convient" se révèlent assez inefficaces pour saisir ce qui est réellement en jeu. Le problème de tout apprenant est d'accéder à la langue cible à partir de la sienne. La langue maternelle est un filtre incontournable et plutôt que de vouloir l'ignorer, il faut l'utiliser comme tremplin, comme clé d'accès au langage. Ce qu'il faut comprendre c'est avant tout pourquoi tel ou tel énoncé est produit, et partant, il faut analyser le statut des composantes de l'énoncé. Voici à la suite un petit test que je proposai dans un ouvrage à destination des professeurs des écoles (in Rosenberger 2003: 88-89) pour les mettre en garde contre une présentation prématurée - et non réfléchie - des «deux présents » de l'anglais ${ }^{34}$, et en particulier de be+ing:

Traduire en anglais les énoncés suivants en tenant compte du contexte dans lequel chacun d'entre eux a été produit :

a. J'habite à Paris, au 14 de la rue Fontaine. (Contexte : renseignement administratif).

b. Je vous reconnais! Vous habitez avec la dame du $6^{\mathrm{e}}$ !

c. Ah, voilà le bus.

d. Dépêche-toi, il y a le bus qui arrive !

e. Aïe! Cela fait mal! (Chez le dentiste!).

f. Arrêtez, vous me faites mal! (Toujours chez le dentiste!).

g. Je ne sais pas quoi faire... Qu'est-ce tu proposes?

h. Mais enfin, qu'est-ce tu me proposes là ?

i. C'est écrit dans le journal, là, regarde : la reine part demain pour l'Australie.

$j$. La reine ne peut pas vous recevoir demain. Elle part pour l'Australie.

k. Si des gens vous disent qu'il y a une meilleure machine à laver, ils mentent.

(Publicité pour Siemens).

Les questions qui se posent devant ces phrases sont les suivantes :

- Comment faire pour savoir lequel des deux Présents choisir pour traduire chacune d'elle?

- De quelle(s) règle(s) est-ce que l'on dispose pour faire le bon choix sans hésiter?

- Est-ce que l'on se sent en mesure de mettre ces règles à la portée des élèves?

On s'aperçoit rapidement que le professeur doit disposer d'outils théoriques pour transposer ces phrases en anglais de façon fiable et que cette réflexion théorique ne pourra concerner les élèves telle quelle. Mais une chose est sûre : on se sera aperçu en faisant l'exercice proposé que les règles traditionnelles ne permettaient pas de se sortir d'affaire. Pire, elles gênent plutôt qu'elles n'aident. Aucune de ces phrases n'est 
pourtant particulièrement difficile ou rare; chacune d'elle est le quotidien de tout un chacun. Voici leur traduction :

Corrigé des dix phrases d'illustration:

a'. I live in Paris...

b'. know you ! You are living with the lady on the sixth floor, aren't you?

c'. Ah, here comes the bus.

d'. Hurry up! The bus is coming !

e'. Ouch! That hurts!

f. Stop it! You are hurting me!

g'. I don't know what to do. What do you suggest?

h'. You what? What are you suggesting ?

i'. It is written in the paper. There, look : the Queen leaves for Australia tomorrow.

j'. The Queen can't see you tomorrow. She is leaving for Australia.

$\mathbf{k}^{\prime}$ If people tell you there is better washing machine, they are lying.

On comprend au vu des traductions qu'il n'y a sans doute pas urgence à vouloir manipuler les deux présents de l'anglais en classe. Chacun de ces énoncés s'inscrit dans un contexte facilement repérable. Le statut des composantes de l'énoncé apparaît très bien dans les deux langues, la différence étant que la langue anglaise offre deux stratégies grammaticales là où le français n'en possède qu'une. Il est clair que dans : «Je vous reconnais! Vous habitez avec la dame du $6^{\mathrm{e}}$ ! le but de l'énoncé n'est pas d'annoncer à l'interlocuteur avec qui il vit mais d'utiliser " habiter avec la dame du $6^{\mathrm{e}}$ " pour corroborer «Je vous reconnais ». La relation vous/habiter avec la dame du $6^{e}$ est de statut repris/non assertif. Outre le statut des composantes des énoncés, l'orientation des énoncés est un paramètre lié. Dans l'énoncé qui suit, une dame évoquait sa situation après que son mari eut été victime d'une maladie très invalidante : I have lost $a$ husband and I am living with a child. Dire ici «I live with a child» signifierait que la dame vit effectivement avec un enfant, ce qui n'est pas le cas ici : elle évoque sa situation difficile et l'état de son mari fait d'elle une maman et non plus une épouse.

On utilisera le petit schéma suivant :

a. I live + with $+a+$ child

b. I am ING[live-with-a-child]

qui est à comprendre de la façon suivante :

2 en a. le point de départ de l'énoncé est le sujet I, le but étant de poser - statut posé/ assertif - la suite. En $\mathbf{b}$. le point de départ de l'énoncé est le bloc en ING. Le but est de partir de ce bloc pour dire où en est le sujet I. L'énoncé a. est "orienté verbe + complément » tandis que $\mathbf{b}$. est « orienté sujet ».

Les exemples qui suivent permettent d'illustrer l'opposition, « simple/be+ing ». En guise de Pratique Raisonnée de la Langue à destination des professeurs, un exercice consistera à replacer chacun de ces énoncés dans un contexte approprié :

I live with my husband and two kids.

I suggest you go there today.

I do not follow. What is wrong with her?

I love that chocolate cake !

Life has not been easy you know. I have lost a husband and I am living with a child.

These people are living on another planet!

You are quite wrong. I am merely suggesting you should go there today, nothing 
more.

Could you be more precise? I am not following you.

Buy one get one free? I am loving it ! 35

Every cigarette is doing you damage ${ }^{36}$

Deux exemples d'insertion dans un contexte :

Jane : Hi Pete ! Please to meet you. I thought we'd met before but I got it wrong.

Pete : Hi Jane! Sarah told me you were coming and that I should with speak with you. Are you from around here?

Jane : I am not actually. I come from Middlesbrough where I live with my husband and two kids. Do you have any children yourself?

I know what they're asking for but a ten per cent increase is out of the question. The firm just can't afford it. These people are living on another planet and will have us in the work house if we listen to them.

Il est capital d'amener les élèves, en commençant par leurs professeurs, à savoir contextualiser un énoncé donné, et donc un outil grammatical donné. Trop d'énoncés décontextualisés ${ }^{37}$ servent d'exemples dans les livres de grammaire.

\section{Conclusion}

Les lauréats d'un concours qui auraient eu à réfléchir de façon sérieuse et motivante au fonctionnement de la langue seraient incontestablement mieux armés face à leurs élèves. C'est après le succès au concours que l'on pourrait entamer la PRL, qui dans mon esprit, concerne en premier chef le professeur. Pour le professeur de collège qui dans l'état actuel des choses n'a aucune consigne quant à l'ordre dans lequel les points de grammaire devraient être abordés, la question de la progression devrait se poser. La deuxième étape dans cette progression serait le choix du type d'énoncés retenu pour présenter tel point de grammaire. Faut-il aborder le présent simple par le truchement des daily routines que tous les manuels d'anglais de collège affectionnent - he gets up at 7 every day - des " caractérisations du sujet »- he likes football - ou au contraire d'énoncés de type "légendes" - écrites ou oralisées ${ }^{38}$ : Charles cuts his cake; Ronaldo waits for the ball, etc. Quelles seront les conséquences du point de départ sur la suite de l'apprentissage ? Comment ce qui aura été présenté à l'étape $\mathrm{n}^{\circ} 1 \mathrm{va}$-t-il conditionner ce qui sera fait à l'étape $n^{\circ} 2, n^{\circ} 3$, etc. ? Dans quel ordre faut-il aborder la présentation des articles $\varnothing, a$, the ? Faut-il ou non enseigner le modal may (May I smoke?) au collège et pourquoi? Quel est le premier énoncé comportant le modal will qui sera le moins dommageable ${ }^{39}$ pour la suite ? Toutes ces questions, rarement - voire jamais - posées dans le cadre de la formation des professeurs d'anglais sont du ressort d'une PRL moderne, qui irait à terme dans le sens de ce qui était préconisé en 1985. Le professeur d'anglais serait alors a skilled technician, et non a casual user (Moulton 1966 : VII). Il n'est pas interdit de penser que plus le point d'entrée est bon et judicieusement choisi, moins les "explications", que la tradition imposait de par une approche sans fondement théorique solide, seront nécessaires. La PRL n'arriverait en classe, de façon réduite mais rigoureuse, qu'après avoir touché le professeur. Pour le lycée, la langue a été mise au second plan. De fait, de par les programmes, un professeur de lycée n'a pas du tout les mêmes objectifs que ses collègues de collège car son enseignement est dominé par le choix de thèmes à aborder. On entendra plus souvent dans les salles des professeurs de lycée "Je ne sais pas quel thème je vais faire avec mes terminales la semaine prochaine » que «Je me demande par quel énoncé je vais introduire (ou revoir) have been-verb-ing. »! Les trois années de lycée ne sont pas gouvernées par la mise en place 
de la langue, mais plutôt par l'étude du racisme, de la violence, de la pollution, etc. et cette approche demeure la marque de fabrique de notre enseignement de l'anglais au lycée. Le contenu des textes de 1987 a, comme celui de 1985, été abandonné sur le bord de la route en ce qui concerne la mise en place de la cohérence grammaticale. À force de redouter le "tout grammaire", de vouloir à tout prix que la grammaire soit minimisée, on a fini par occulter le fait que l'épine dorsale d'une langue ne pouvait pas être abordée par le biais d'une approche dite actionnelle. Le collège effleure la langue et le lycée considère que l'essentiel est "vu », pour aller droit aux descriptions de documents iconographiques et autres données culturelles. Est-ce trop demander aujourd'hui encore de vouloir que les professeurs reçoivent une formation linguistique adéquate?

\section{BIBLIOGRAPHIE}

\section{Sources primaires}

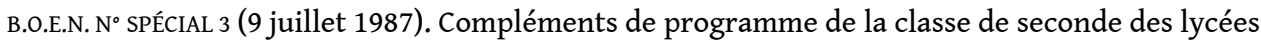
(Arrêtés des 14 mars et 30 juin 1986). Paris : BO/CNDP. 19-26.

B.O.E.N. $\mathrm{N}^{\circ} 7$ (3 octobre 2002). Programme d'enseignement de l'anglais, classe de seconde générale et technologique. Paris : BO/CNDP.

CARPENTIER-FIALIP, Pierre \& Madeleine (1931). L'anglais vivant. Classe de $6^{\mathrm{e}}$. Paris : Hachette.

GIRARD, Denis (1978). Grammaire raisonnée de l'anglais. Paris : Hachette Éducation.

MARTINA, Claudine (2000). La grammaire de l'anglais au collège. Paris : Belin.

MINISTÈRE DE L'ÉDUCATION NATIONALE (1985). Collèges. Programmes et instructions. Paris : BO/CNDP.

PERSEC, Sylvie ; avec la collaboration de Hélène Pech-Gourg (1994). Grammaire raisonnée au lycée : compétence linguistique en anglais. Paris : Ophrys.

\section{Sources secondaires}

ADAMCZEWSKI, Henri (1974). « BE + ING Revisited ». In Stephen Pit Corder \& Eddy Roulet (eds.), Linguistic Insights in Applied Linguistics. Bruxelles : AIMAV et Paris : Didier. 45-75.

ADAMCZEWSKI (1975). « Esquisse d'une théorie de DO ». In Stephen Pit Corder \& Eddy Roulet (eds.), Some Implications in Linguistics Theory for Applied Linguistics. Bruxelles : AIMAV, et Paris : Didier. 47-55.

ADAMCZEWSKI (1978). BE + ING dans la grammaire de l'anglais contemporain. Thèse d'état. Paris : Honoré Champion. En ligne : [http://www.crelingua.fr/index.php? option $=$ com_content $\&$ view $=$ article $\& i d=30$ :being-dans-la-grammaire-de-langlaiscontemporain\&catid $=1$ :ouvrages\&Itemid $=2]$. 
ADAMCZEWSKI (1982). Grammaire linguistique de l'anglais. Paris : Armand Colin.

ADAMCZEWSKI (2016). «BE + ING» : transcription d'un cours de licence enregistré pour

l'enseignement par correspondance à la fin des années 1970. Publications du Crelingua [http:// www.crelingua.fr/].

ADAMCZEWSKI, Henri \& GABILAN, Jean-Pierre (1993). Les clés de la grammaire anglaise. Paris : Armand Colin.

GABILAN, Jean-Pierre (1995). « À propos des manuels d'anglais ». La Tribune Internationale des Langues Vivantes 17/3.

GABILAN (2006). Grammaire expliquée de l'anglais. Paris : Ellipses.

GABILAN (2007). « Le temps ne fait rien à l'affaire ». Les Langues Modernes 2, 21-31.

GABILAN (2008). « Comprendre et enseigner be+ing ». En ligne : [http://cle.ens-lyon.fr/anglais/

langue/linguistique/comprendre-et-enseigner-be-ing-].

MOULTON, william (1966). A Linguistic Guide to language Learning. New York : Modern Language

Association of America.

ROSENBERGER, Sophie (dir.) (2003) : Guide pour enseigner l'anglais à l'école élémentaire. Paris : Retz, 88-89.

SAUSSURE, Ferdinand de (1949). Cours de Linguistique Générale. Paris : Payot.

\section{NOTES}

1. En ligne : [http://media.education.gouv.fr/file/21/4/5214.pdf].

2. Ce qui ne surprend pas. La langue orale reste en effet le parent pauvre de l'enseignement de l'anglais ; absente même du principal concours de recrutement des professeurs d'anglais (CAPES) puisque ce concours n'évalue pas la prononciation des candidats de façon sérieuse par le biais d'une épreuve spécifique. Il n'est pas étonnant qu'au collège, puis au lycée, l'anglais oral des anglophones reste un mystère, tant en compréhension qu'en production.

3. L'anglais est également présent à l'École élémentaire. Même si la grammaire ne semble pas y occuper une place prépondérante, il y a grammaire dès lors que l'on expose des enfants à des énoncés et qu'on leur demande d'en produire à leur tour.

4. Arrêté du 14 novembre 1985 pour le collège.

5. Il n'est pas assuré que plus de trente ans plus tard ces mêmes recommandations seraient comprises.

6. Au moment où les textes de 1985 ont été publiés, les ouvrages universitaires de références étaient les suivants. Dans une perspective «traditionnelle»: André Tellier, Cours de grammaire anglaise. Paris: Sedes, 1967 ; Serge Berland Delépine, Grammaire anglaise de l'étudiant. Paris : Ophrys, 1971 ; en lien avec la linguistique : Henri Adamczewski, Grammaire linguistique de l'anglais. Paris: A. Colin, 1982. Quant aux articles scientifiques des publications spécialisées, ils n'ont jamais franchi la porte des collèges et des lycées.

7. On songe ici à Saussure (1949: 159) : «[...] la langue est un système dont tous les termes sont solidaires et où la valeur de l'un ne résulte que de la présence simultanée des autres [...] ».

8. L'épreuve de grammaire du CAPES d'anglais - Certificat d'Aptitude au Professorat de l'Enseignement Secondaire - consistait à faire une analyse grammaticale de trois éléments soulignés dans un texte (une nouvelle inédite) faisant l'objet d'une présentation orale. L'exercice 
de grammaire ne prenait qu'une dizaine de minutes - dix minutes pour une carrière de quatre décennies.

9. Contrairement à la situation qui prévalait encore en 1987, les candidats au CAPES, tel qu'il se présente maintenant, ne connaissent pas la note qui leur a été attribuée à l'épreuve dite des faits de langue, celle-ci faisant partie d'un ensemble dont seul le jury a le détail.

10. (1994). Wings $6^{e}$. Anglais pour le collège, Belin.

11. Présent dit simple : He drinks; et Présent be+ing : He is drinking.

12. Éditions Belin.

13. (1925-2002). Il fut inspecteur général d'anglais et auteur de manuels, de grammaires et de dictionnaires d'anglais.

14. Ce qui différencie this de that est invariablement expliqué par la distance qui séparerait l'énonciateur de l'objet désigné. Les énoncés authentiques du quotidien invalident cette explication.

15. Une présentation identique fut reprise pour les textes publiés entre 1995 et 1998.

16. Il semble que cette opération porte un nom : la narcotisation du sigle.

17. Il s'agissait de la leçon à laquelle assistaient un inspecteur et les conseillers pédagogiques et à l'issue de laquelle le professeur stagiaire était soit reçu et titularisé soit recalé.

18. Il était écrit à ce propos que les textes de 1987 «feraient date dans l'histoire de l'enseignement de l'anglais » sur ce point. Force est de constater qu'il ne suffit pas de publier de beaux textes pour qu'ils soient appliqués à la lettre.

19. Ce ne fut qu'un effet d'annonce. La phonologie au sens fort est restée absente des classes d'anglais.

20. En voici les titres: Wings (Belin), off we go (Bordas), Live (Didier), The New Apple Pie (Hachette), Gulliver Street (Hatier), No Problem (Longman), Action (Nathan).

21. Cf. Gabilan (1995) pour une analyse détaillée.

22. La quatrième de couverture mentionne ce qui suit: "Comment faire découvrir à l'élève le fonctionnement de la cohérence de l'anglais. "

23. C'est par exemple la position de Michel Viel à la fin d'un entretien publié dans L'US MAG supplément au n 577 du 25 octobre 2002 - à l'occasion de la parution en 2002 de Variations sur la linguistique, d'Antoine Culioli : "Pour étudier la linguistique d'une langue il faut déjà connaître la langue et non le contraire. ». En ligne : [https://www.snes.edu/IMG/pdf/us577bis.pdf].

24. Cf. Adamczewski 1973 pour une remise en cause des analyses traditionnelles de be+ing.

25. Mission Académique de Formation des Personnels Enseignants; Centre Régional de Documentation Pédagogique.

26. Cadre européen commun de référence pour les langues.

27. Citons ici Henri Adamczewski (1982:9) : «Le premier chapitre d'une grammaire ne peut en aucun cas devoir sa qualité de premier au sens où il serait placé avant le deuxième. Il doit être premier au sens fort, c'est-à-dire qu'il doit conditionner la suite, constituer la base sur laquelle on va construire les autres chapitres. »

28. Gustave Guillaume préférait article « définissant ».

29. Le mot «temps » n'est bien entendu pas satisfaisant car il donne à penser que les «temps » portés par les verbes auraient un rapport direct avec l'extralinguistique. Le temps appelé « présent » peut très bien affecter un énoncé évoquant l'avenir ou le révolu.

30. Cf. Adamczewski $(2016: 10,12)$.

31. Cf. Adamczewski (1974) pour une analyse complète du rôle de DO.

32. La place manque ici pour donner des analyses détaillées. On se reportera à Adamczewski 1982, Adamczeswki \& Gabilan 1993, Gabilan 2006 pour les explications de fonctionnement de ces paires minimales qui reposent toutes sur le vecteur posé-assertif/repris-non assertif.
33. En
ligne :
[http://media.devenirenseignant.gouv.fr/file/ext/21/2/

RJ_2017_CAPES_externe_ANGLAIS_796212.pdf]. 
34. Présent « simple » et Présent «be+ing ».

35. «I'm loving it. » fut pendant plusieurs années le slogan utilisé par McDonald. Il était à l'origine la conclusion de toute une série de petits films publicitaires et l'énoncé arrivait en guise conclusion - et ne signifiait nullement l'expression d'une déclaration d'amour. Nombre de professeurs d'anglais pour lesquels le verbe love « était incompatible avec be+ing » furent quelque peu décontenancés par cet énoncé qui connut un succès planétaire.

36. Cet énoncé était la conclusion d'une série d'images choc utilisées pour une campagne antitabac. Tout recours à la forme « simple » est ici impossible.

37. On fera bien la différence entre un énoncé produit en contexte authentique et une phrase, fabrication sans contexte et grammaticalement inexploitable.

38. Cf. Gabilan (2007 et 2017) sur ce point.

39. Si l'on dit que will = futur, comme on le fait majoritairement, le fonctionnement du modal will reste à jamais opaque et dans un énoncé aussi banal que « I will call you when he gets back from work » la non présence de will après when demeure un mystère grammatical.

\section{RÉSUMÉS}

Apparu en France en 1985 dans les instructions officielles régissant l'enseignement de l'anglais au collège, le concept de Pratique Raisonnée de la Langue augurait de la mise en place d'un enseignement de la langue dans lequel la réflexion grammaticale aurait été un élément clé. Mais publiées à un moment inopportun, à un moment où les professeurs n'avaient eux-mêmes pas été formés à une grammaire scientifique, les instructions officielles de 1985 sont restées «lettre mortes ». La Pratique Raisonnée de la Langue a été oubliée et de fait la grammaire - et la phonologie - sont aujourd'hui négligées, et ce dès le concours de recrutement. Qu'entendait-on en 1985 par "grands principes de fonctionnement de l'anglais"? Quels sont-ils? Quelles stratégies peut-on proposer aux professeurs pour qu'ils puissent rendre intelligibles à leurs élèves les agencements grammaticaux des énoncés du quotidien?

In 1985 a new set of formal instructions for the teaching of English in France in secondary schools had introduced a concept similar to that of language awareness urging teachers to help students understand how English truly works and to do so to help them discover the existence of the "set of great principles that make English work". But at the time, very few teachers, to say the least, knew what this was all about. Traditional grammar teaching with its rules and inevitable exceptions were still strong. Language awareness was soon forgotten and it is no secret that up to this day grammar and pronunciation have always been neglected and neglected to the point that neither is even a key element in the competitive exam one has to take to become a teacher. What were these "great principles" supposed to be back in 1985 ? What are they exactly? What can be taught to teachers while being trained so that they can help their students cope with everyday sentences traditional grammar teaching has always failed to explain? 
INDEX

Mots-clés : Pratique Raisonnée de la Langue, grands principes de fonctionnent, statut assertif, statut non assertif, grammaire

Keywords : awareness of language, great principles making languages work, assertive status, non assertive status, grammar

\section{AUTEUR}

JEAN-PIERRE GABILAN

Université de Savoie, laboratoire LLSETI

jean-pierre.gabilan@univ-savoie.fr 